\title{
Dépendance socioculturelle des connaissances locales des usages de Isoberlinia spp. au Moyen-Bénin, Afrique de l'Ouest
}

Sessi Gilles Christian Adjahossou ${ }^{1,2}$ Dèhouégnon Thierry HouÉHANOU 2, 3, 4 Mireille Toyı ${ }^{2}$

Valère Kolawolé SALAKO ${ }^{4}$

Carlos Cédric AHOYO ${ }^{2}$

Paolo LESSE ${ }^{2}$

Brice TENTE $^{5}$

Marcel Roland Benjamin HouINATO

${ }^{1}$ Direction générale des forêts et des ressources naturelles (DGFRN) BP 393, Cotonou

République du Bénin

2 Université d’Abomey-Calavi (UAC) Faculté des sciences agronomiques (FSA)

Laboratoire d'écologie appliquée (LEA) 01 BP 526, Cotonou

République du Bénin

${ }^{3}$ Université de Parakou

Faculté d'agronomie

Laboratoire d'écologie, de botanique et de biologie végétale

03 BP 125, Parakou

République du Bénin

${ }^{4}$ Université d'Abomey-Calavi (UAC) Faculté des sciences agronomiques (FSA)

Laboratoire de biomathématiques et d'estimations forestières (LABEF) 04 BP 1525, Cotonou République du Bénin

${ }^{5}$ Université d'Abomey-Calavi Département de géographie République du Bénin

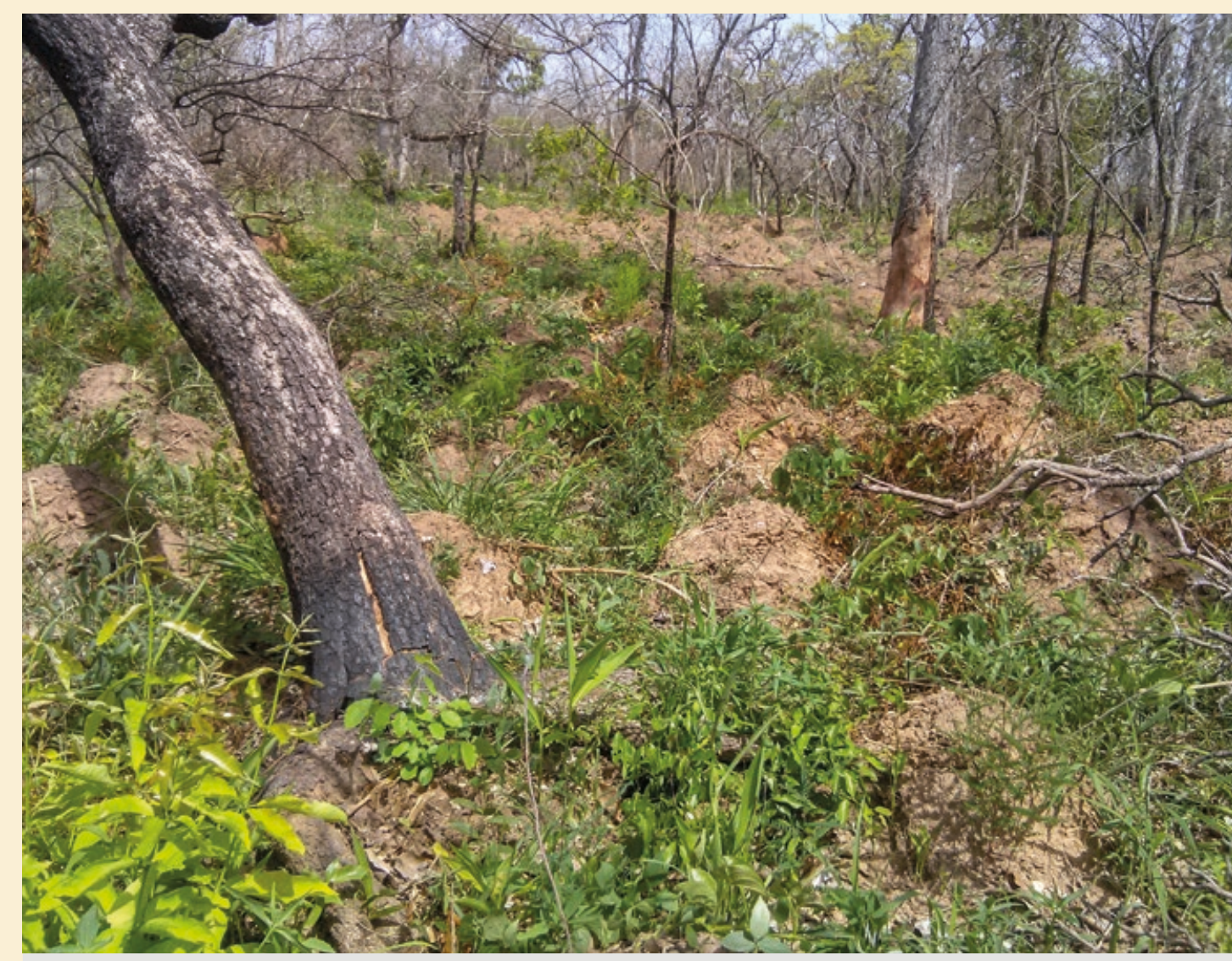

Auteur correspondant / Corresponding author:

Sessi Gilles Christian Adjahossou christdiord@gmail.com

Photo 1.

Buttes d'igname au sein d'un peuplement à Isoberlinia spp. récemment défriché dans la forêt classée de Wari-Maro. Photo S. G. C. Adjahossou.

Doi : 10.19182/bft2019.339.a31702 - Droit d'auteur ( 2018 , Bois et Forêts des Tropiques - @ Cirad - Date de soumission : 12 février 2018 ; date d'acceptation : 30 octobre 2018 ; date de publication : 15 janvier 2019.

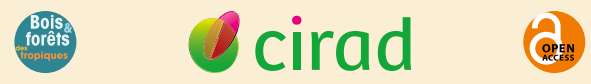

(c) $(1) \Theta$
Citer l'article / To cite the article

Adjahossou S. G. C., Houéhanou D. T., Toyi M., Salako V. K., Ahoyo C. C., Lesse P., Tente B., Houinato M. R. B., 2019. Dépendance socioculturelle des connaissances locales des usages de Isoberlinia spp. au MoyenBénin, Afrique de l'Ouest. Bois et Forêts des Tropiques, 339 : 33-43. Doi : https://doi.org/10.19182/bft2019.339.a31702 


\section{S. G. C. Adjahossou, D. T. Houéhanou, M. ToY, V. K. Salako, C. C. AhoYo, P. Lesse, B. TENTE, M. R. B. Houinato}

\section{ABSTRACT}

\section{RESUMEN}

\section{Sociocultural reliance on local knowledge of the uses of Isoberlinia spp. in Central Benin, West Africa}

\section{Dépendance socioculturelle des connaissances locales des usages de Isoberlinia spp. au Moyen-Bénin, Afrique de l'Ouest}

Isoberlinia doka Craib \& Stapf et Isoberlinia tomentosa (Harms) Craib \& Stapf sont deux arbres originaires d'Afrique. Autrefois peu convoités, ils revêtent actuellement une utilité avérée pour les populations locales. Au Moyen-Bénin, l'intégration des savoirs traditionnels relatifs au genre Isoberlinia dans les stratégies de développement local demeure importante mais peu documentée. L'objectif de cette étude était d'évaluer, d'une part, les connaissances endogènes relatives aux utilisations des deux essences et, d'autre part, l'effet de cinq facteurs socioculturels ainsi que leurs interactions sur la valeur d'usage des deux essences dans le Moyen-Bénin. Des enquêtes ethnobotaniques ont été conduites auprès de 480 informateurs répartis dans huit groupes socioculturels. La fréquence relative de citation (FRC) et la valeur d'usage (VU) ont été calculées et analysées en utilisant respectivement une analyse en composantes principales (ACP) et des modèles linéaires généralisés (GLM) basés sur la distribution de Poisson. Les Mahi et Nago utilisent plus I. doka comme bois de charpente; les Dendi et Holli utilisent plus I. tomentosa comme bois d'œuvre. Les modèles simples à un facteur montrent que, parmi les facteurs testés, le groupe socioculturel détermine les variations de l'usage des deux essences, et l'activité professionnelle influence aussi l'usage de I. tomentosa. L'évaluation de l'effet simultané des cinq facteurs sociaux et de leur interaction dans un même modèle multiple montre que les différences de la valeur d'usage de I. tomentosa entre les groupes socioculturels peuvent être amplifiées par l'activité professionnelle. Par ailleurs, pour I. doka, le modèle incluant uniquement le groupe socioculturel est celui le plus parcimonieux. Les politiques de gestion durable des deux essences devraient intégrer au premier plan les considérations socioculturelles, auxquelles s'ajoutent les appartenances professionnelles et, dans une moindre mesure, la taille du ménage pour I. tomentosa.

Mots-clés : savoirs traditionnels, facteurs sociaux, patron d'usage, intensité d'utilisation, interactions, Bénin.
Isoberlinia doka Craib \& Stapf and Isoberlinia tomentosa (Harms) Craib \& Stapf are both native African tree species. Although considered of little value in the past, they are now proving useful to local populations. In Central Benin, traditional knowledge on the genus Isoberlinia is widely applied in local development strategies, but little documented. This study aimed to assess (i) endogenous knowledge on uses of the two species and (ii) the effects of five sociocultural factors, and their interactions, on the use value of the two species in Central Benin. Ethnobotanical surveys were conducted with 480 respondents divided into eight sociocultural groups. Relative frequency of citation (RFC) and use value (UV) were calculated and analysed into their principal components (PCA), and generalised linear models (GLM) were produced based on Poisson distributions. The Mahi and Nago people make more use of $I$. doka as roof timbers; the Dendi and Holli make more use of $I$. tomentosa for building. Simple single-factor models show that among the factors tested, the sociocultural group determines variations in the use of the two species, while professional activities also influence the use of $I$. tomentosa. Our assessment of the simultaneous effects of the five social factors and their interactions in a single multiple model shows that the differences in use value of $I$. tomentosa among the sociocultural groups can be amplified by professional activities. Furthermore, for I. doka, the model including sociocultural groups only shows the fewest uses. In developing policies to manage the two species sustainably, sociocultural factors must therefore be a primary consideration, followed by professional categories and, for I. tomentosa and to a lesser extent, household size.

Keywords: Traditional knowledge, sociocultural factors, use pattern, use intensity, interactions, Benin.

\section{Dependencia sociocultural de los conocimientos locales de los usos de Isoberlinia spp. en el Benín Medio, África Occidental}

Isoberlinia doka Craib \& Stapf e Isoberlinia tomentosa (Harms) Craib \& Stapf son dos árboles originarios de África. Antaño poco codiciados, actualmente presentan una utilidad demostrada para las poblaciones locales. En el Benín Medio la integración de los saberes tradicionales relativos al género Isoberlinia en las estrategias de desarrollo local continúa siendo importante pero poco documentada. El objetivo de este estudio es evaluar (i) los conocimientos endógenos relativos a los usos de las dos especies y (ii) el efecto de cinco factores socioculturales, así como sus interacciones sobre el valor de uso de las dos especies en el Benín Medio. Se han realizado encuestas etnobotánicas a unos 480 informadores pertenecientes a ocho grupos socioculturales. La frecuencia relativa de citación (FRC) y el valor de uso (VU) se han calculado y analizado utilizando respectivamente un análisis de componentes principales (ACP) y modelos lineales generalizados (GLM) basados en la distribución de Poisson. Los Mahi y Nago utilizan más I. doka como madera estructural; los Dendi y Holli utilizan más I. tomentosa como madera de obra. Los modelos simples de un factor muestran que, entre los factores probados, el grupo sociocultural determina las variaciones del uso de las dos especies, y la actividad profesional influye también en el uso de I. tomentosa. La evaluación del efecto simultáneo de los cinco factores sociales y de su interacción en un mismo modelo múltiple muestra que las diferencias del valor de uso de I. tomentosa entre los grupos socioculturales pueden ser amplificadas por la actividad profesional. Además, para I. doka, el modelo que incluye únicamente el grupo sociocultural es el más parsimonioso. Las políticas de gestión sostenible de las dos especies deberían integrar en primer lugar las consideraciones socioculturales, a las que se añaden las profesionales, $y$, en menor medida, las dimensiones de los hogares para I. tomentosa.

Palabras clave: saberes tradicionales, factores sociales, patrón de uso, intensidad de uso, interacciones, Benín. 


\section{Introduction}

Originaire d'Afrique et appartenant à la sous-famille des Caesalpinoideae (Akouègninou et al., 2006), le genre Isoberlinia, autrefois peu convoité, revêt de nos jours une utilité avérée compte tenu de la dégradation et de l'appauvrissement des forêts denses humides et denses sèches en essences de valeur (Dourma, 2011 ; Louppe, 2012). Identifiées comme des espèces ligneuses susceptibles d'être domestiquées en région tropicale, un intérêt croissant leur est désormais accordé. Cet intérêt vise leur utilisation comme bois énergie, bois de service, bois d'œuvre et phytomédicament pour satisfaire les besoins des populations autochtones (Bellefontaine et al., 2015).

Dans les sociétés africaines, les populations indigènes dépendent étroitement des ressources végétales ligneuses et s'appuient sur leurs connaissances pour faire face aux problèmes quotidiens de pauvreté, de faim et d'insécurité alimentaire (Fandohan et al., 2015 ; Yaoitcha et al., 2015). La dépendance des populations locales africaines vis-à-vis des ressources ligneuses en milieu naturel pourrait dès lors être une menace pour la survie de ces espèces végétales soumises aux pressions humaines (Adjahossou et al., 2016, 2018). De nombreuses études conduites au Bénin (Assogbadjo et al., 2008 ; Fandohan et al., 2010 ; Houéhanou et al., 2011) et ailleurs (Dourma, 2011) se sont intéressé à concilier la subsistance des populations locales avec l'exploitation des ressources ligneuses locales en voie de disparition, en se focalisant sur les aspects ethnobotaniques et socio-économiques.

L'étude des connaissances locales s'avère impérieuse pour capitaliser les savoirs traditionnels dans les stratégies de gestion des ressources naturelles ligneuses de valeur (Yaoitcha et al., 2015 ; Akpona et al., 2017). De même, les connaissances sur la manière dont les facteurs sociaux influencent l'exploitation d'une ressource apparaissent capitales pour envisager une exploitation durable avec les acteurs spécifiques concernés au niveau local. Face à cette problématique d'interaction des facteurs sociaux avec les connaissances sur les ressources locales, plusieurs travaux ont impliqué des facteurs sociaux tels le groupe socioculturel, l'âge ainsi que la taille du ménage, dans l'analyse des savoirs traditionnels pour une gestion judicieuse des ressources locales (Gavin et Anderson, 2017). De plus, certains travaux ont observé que d'autres caractéristiques individuelles comme les responsabilités au niveau familial, la profession, les aptitudes et les capacités intellectuelles pouvaient tout autant influencer la connaissance des ressources ligneuses et leur utilisation au sein d'un groupe (Lougbégnon et al., 2011 ; Fandohan et al., 2017).

En Afrique, la littérature abonde sur les travaux relatifs à la description des structures de peuplements de Isoberlinia dans différentes forêts (Bationo et al., 2005 ; Dourma, 2011), voire les usages ethnobotaniques courants (Dourma, 2011 ; Louppe, 2012). Au Bénin, afin de mieux orienter les opérations de restauration des ligneux autochtones au cours de cette dernière décennie, des recherches se sont focalisées sur l'importance socioculturelle des plantes en prenant en compte les réalités perçues par les populations locales (Yaoitcha et al., 2015 ; Assogba et al., 2017).
À ce titre, les connaissances en ethnoécologie pour l'intégration des espèces du genre Isoberlinia dans les stratégies d'utilisation durable et de diversification de la production sylvicole pour la conservation demeurent insuffisamment répandues et documentées. Aussi la présente étude visaitelle à documenter les connaissances locales sur les usages du genre Isoberlinia au Moyen-Bénin, et à évaluer l'effet isolé et simultané de cinq facteurs sociaux (groupe socioculturel, âge, catégorie socioprofessionnelle, niveau d'instruction, taille du ménage) ainsi que leurs interactions sur l'intensité d'utilisation exprimée sous forme de valeur d'usage totale (VU) des deux essences dans le Moyen-Bénin.

\section{Matériels et méthodes}

\section{Milieu d’étude}

Le milieu d'étude (figure 1) s'étend sur une superficie de $29888 \mathrm{~km}^{2}$ et se situe dans la zone de transition entre les climats subéquatorial du Sud et tropical humide du Nord (Yabi, 2007 ; Oloukoï, 2012). Ces dernières années, la tendance pluviométrique est unimodale à base large et les hauteurs pluviométriques annuelles oscillent entre 900 et $1300 \mathrm{~mm}$. L'analyse du paysage végétal du Moyen-Bénin montre une anthropisation poussée des formations naturelles par, notamment, l'agriculture et l'exploitation forestière. Plusieurs groupes socioculturels peuplent la zone d'étude avec un taux d'accroissement de la population de 3,61 \% entre 2002 et 2013 (INSAE, 2016). Il s'agit des Fon,

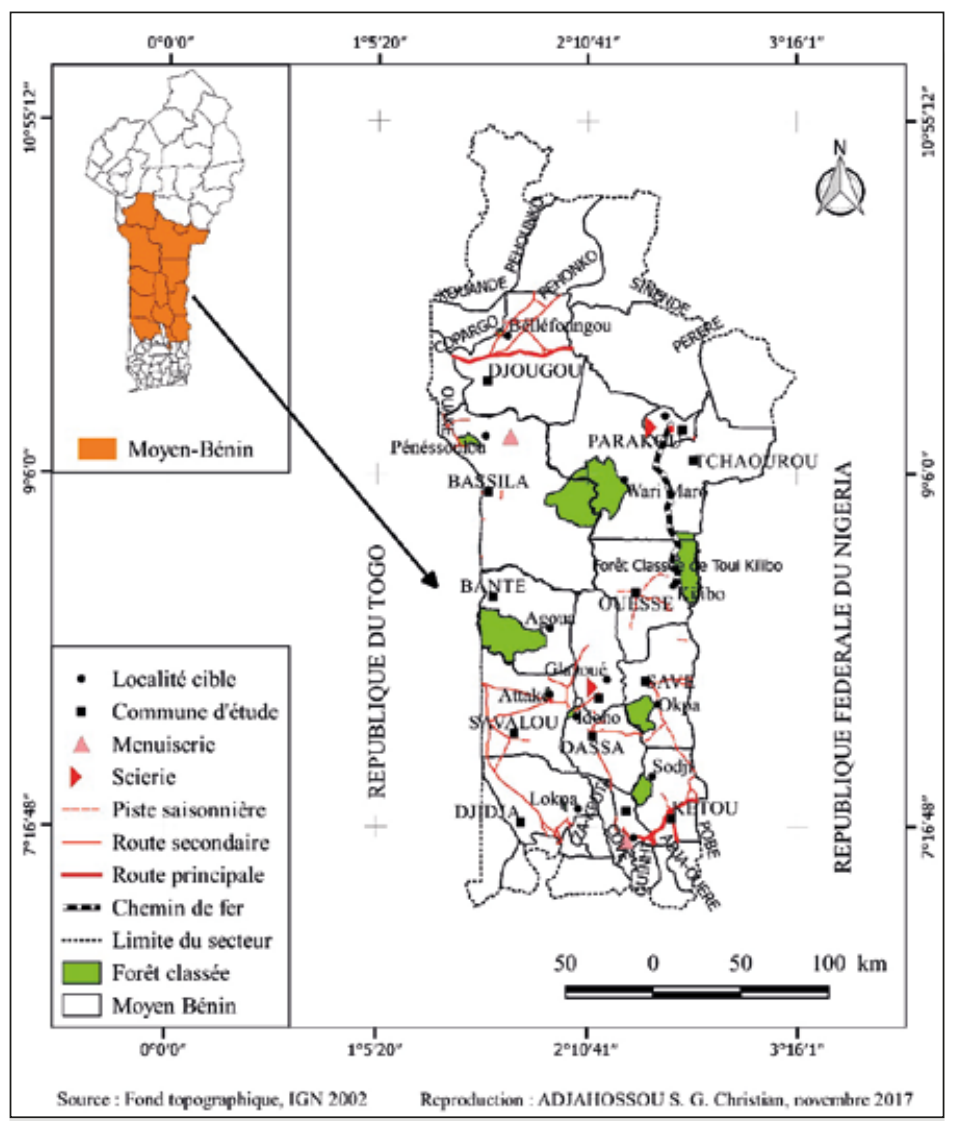

Figure 1.

Localisation du milieu d’étude. 
Yoruba, Mahi, Nago, Anii, Kotokoli, Dendi, Bariba, Holli et Biali. Près de $79 \%$ se consacrent essentiellement à l'agriculture, les autres intervenant dans la commercialisation du charbon de bois, l'artisanat, l'exploitation forestière, la menuiserie et la transformation de produits forestiers non ligneux (INSAE, 2016).

\section{Échantillonnage et collecte des données}

La sélection des informateurs a été conduite de façon aléatoire et a porté sur huit groupes socioculturels (Mahi, Nago, Anii, Kotokoli, Dendi, Bariba, Holli et Fon) en tenant compte de la distribution des groupes socioculturels dans le milieu d'étude, de la proximité des villages par rapport aux forêts classées et de la disponibilité des essences ligneuses cibles dans les terroirs.

Inspiré des études réalisées en ethnoécologie au Bénin (Ahoyo et al., 2018) et au Pérou (Phillips et Gentry, 1993), l'indice de connaissance (IK) relatif aux usages du genre Isoberlinia au sein d'un groupe socioculturel pris au hasard dans le Moyen-Bénin est le rapport du nombre d'usages cités $(R)$ par le groupe socioculturel et du nombre (n) de personnes enquêtées dans le même groupe :

$$
I K=\frac{R}{n}
$$

Pour que le seuil de connaissance $(I K)$ maximal critique soit estimé, il faut que les valeurs critiques de $R$ et $n$ soient maximales. Dans la présente étude, la valeur maximale de $R$ est constante et égale à quatre (charpente, œuvre, énergie, et à usage médicinal). Pour calculer $n$, constante maximale critique équivalente au nombre de personnes à interviewer par groupe socioculturel, une enquête exploratoire a été effectuée auprès de 100 personnes choisies au hasard au sein d'un groupe socioculturel également sélectionné de façon aléatoire (Mahi) parmi les huit groupes socioculturels cibles afin de déterminer la proportion $p$ de répondants connaissant les quatre usages cibles. Le nombre $n$ d'enquêtés par groupe socioculturel a été ensuite estimé comme suit (Dagnelie, 1998 ; Levy et Lemeshow, 2008) :

$n=\frac{U_{(1-\alpha / 2)}^{2} p(1-p)}{d^{2}}$

avec $n$ la valeur maximale critique du nombre de personnes à interviewer par groupe socioculturel ; la proportion d'informateurs qui utilisent l'espèce cible et déterminée lors de la phase exploratoire $(p=0,90) ; U_{1-\alpha / 2}$ la valeur de la statistique de la loi normale à la valeur de probabilité $1-\alpha / 2$ avec $\alpha=5 \%$, égale à 1,96; d la marge d'erreur de l'estimation fixée à une valeur de $8 \%$.

L'application de cette formule donne $n=60,025$, soit 60 informateurs.

Pour un groupe socioculturel cible aléatoirement choisi (Mahi), la variable $n$ équivaut à 60 personnes, avec huit groupes socioculturels; la taille de l'échantillon est portée à $8 \mathrm{n}$, soit 480 informateurs de différentes catégories d'activités socioprofessionnelles (scieur, menuisier, charbonnier et agriculteur) rencontrées dans la zone d'étude. Avec l'aide des autorités locales, les données ethnobotaniques ont été collectées auprès des riverains de certaines forêts classées du milieu d'étude. Avec l'assistance d'un traducteur local au besoin, chaque enquêté a été soumis à un entretien semi-structuré sur la base d'un questionnaire.

\section{Traitement et analyses statistiques}

L'analyse des données a été conduite en recourant à cinq facteurs sociaux considérés dans la présente étude (tableau I) : le groupe socioculturel, l'âge, la catégorie socioprofessionnelle, le niveau d'instruction et la taille du ménage (Souto et Ticktin, 2012 ; Assogba et al., 2017 ; Etongo et al., 2017 ; Sinasson et al., 2017). En se raccordant aux études

Tableau I.

Répartition des personnes enquêtées suivant les facteurs sociaux.

\begin{tabular}{|c|c|c|c|c|c|c|c|c|c|c|}
\hline \multicolumn{2}{|l|}{ Groupe socioculturel } & \multirow{2}{*}{$\begin{array}{c}\text { Mahi } \\
18\end{array}$} & \multirow{2}{*}{$\begin{array}{c}\text { Nago } \\
37\end{array}$} & \multirow{2}{*}{$\begin{array}{l}\text { Anii } \\
44\end{array}$} & \multirow{2}{*}{$\begin{array}{c}\text { Kotokoli } \\
16 \\
\end{array}$} & \multirow{2}{*}{$\begin{array}{c}\text { Dendi } \\
42\end{array}$} & \multirow{2}{*}{$\begin{array}{c}\text { Bariba } \\
46\end{array}$} & \multirow{2}{*}{$\begin{array}{l}\text { Holli } \\
40 \\
\end{array}$} & \multirow{2}{*}{$\begin{array}{l}\text { Fon } \\
45\end{array}$} & \multirow{2}{*}{$\begin{array}{l}\text { Total } \\
\\
298\end{array}$} \\
\hline Âge & Jeune & & & & & & & & & \\
\hline & Adulte & 36 & 11 & 10 & 14 & 13 & 13 & 8 & 10 & 105 \\
\hline & Âgé & 6 & 12 & 6 & 30 & 5 & 1 & 12 & 5 & 77 \\
\hline & Total & 60 & 60 & 60 & 60 & 60 & 60 & 60 & 60 & 480 \\
\hline \multirow[t]{5}{*}{ Profession } & Scieur & 17 & 15 & 20 & 15 & 38 & 20 & 28 & 23 & 176 \\
\hline & Menuisier & 15 & 22 & 20 & 17 & 16 & 21 & 16 & 7 & 134 \\
\hline & Agriculteur & 22 & 16 & 19 & 18 & 6 & 3 & 15 & 21 & 121 \\
\hline & Charbonnier & 6 & 7 & 1 & 10 & 0 & 16 & 1 & 9 & 50 \\
\hline & Total & 60 & 60 & 60 & 60 & 60 & 60 & 60 & 60 & 480 \\
\hline \multirow[t]{3}{*}{ Niveau d'instruction } & Non instruit & 51 & 56 & 51 & 48 & 47 & 48 & 57 & 45 & 403 \\
\hline & Instruit & 9 & 4 & 9 & 12 & 13 & 12 & 3 & 15 & 77 \\
\hline & Total & 60 & 60 & 60 & 60 & 60 & 60 & 60 & 60 & 480 \\
\hline \multirow[t]{3}{*}{ Taille du ménage } & Petit ménage & 49 & 45 & 49 & 33 & 44 & 19 & 31 & 48 & 318 \\
\hline & Grand ménage & 11 & 15 & 11 & 27 & 16 & 41 & 29 & 12 & 162 \\
\hline & Total & 60 & 60 & 60 & 60 & 60 & 60 & 60 & 60 & 480 \\
\hline
\end{tabular}


ethnobotaniques sus-référencées effectuées au Bénin, il a été défini trois classes d’âge ( $\leq 40$ ans, 41-50 ans, > 50 ans), deux niveaux d'instruction (non instruit et instruit) et deux tailles du ménage (petit ménage et grand ménage). Les noninstruits sont ceux qui ne savent ni lire ni écrire. Les petits ménages ont au plus cinq membres à charge.

Deux indices ethnobotaniques, la fréquence relative de citation (FRC) et la valeur d'usage (VU), ont été calculés.

La FRC a été calculée par groupe socioculturel et par modalité de chaque facteur social examiné (âge, catégorie socioprofessionnelle, niveau d'instruction et taille du ménage) selon la formule :

FRC $=$ m / N (Tardío et Pardo-de-Santayana, 2008 ; Houéhanou et al., 2011)

avec $m$ le nombre de personnes ayant cité l'information dans le groupe cible; $\mathrm{N}$ le nombre total de personnes interviewées dans le groupe cible.

Seules les FRC significatives (FRC $\geq 5 \%$ ) (Gouwakinnou et al., 2011 ; Houéhanou et al., 2016 ; Fandohan et al., 2017) ont été prises en compte.

Une analyse en composantes principales (ACP) sur les données de FRC de chaque usage par groupe socioculturel a permis de déterminer les patrons d'usage de chacune des deux espèces en fonction des groupes socioculturels.

Des diagrammes en barre des moyennes de FRC surmontés de barres d'erreur ont été générés pour illustrer l'importance relative des quatre utilisations suivant les facteurs socioculturels (âge, profession, niveau d'instruction et taille du ménage).

Pour évaluer l'intensité des usages en relation avec les facteurs sociaux investigués, la valeur d'usage totale (VU) a été calculée pour chaque essence. La VU est la moyenne des nombres d'usages assignés par les enquêtés à chaque essence :
VU $=\sum_{i=1}^{n} U i / N($ Phillips et Gentry, 1993 ; Fandohan et al., 2017)

avec $U_{i}$ : nombre d'usages mentionnés par l'informateur i du groupe social, variant de 0 à 4 ; $\mathrm{N}$ : nombre total d'informateurs interviewés par modalité de chaque facteur.

La valeur d'usage calculée suit une distribution de Poisson puisqu'il s'agit de la moyenne d'une variable de comptage (Salako et al., 2018). Ainsi, un modèle linéaire généralisé (GLM) de la famille des lois de Poisson a été réalisé pour tester l'effet séparé de chacun des cinq facteurs sociaux sur la VU. Ces modèles étaient donc simples, à un facteur. Toutefois, les effets principaux tels qu'analysés peuvent cacher des effets synergiques de plusieurs facteurs qui peuvent être en revanche captés dans des modèles multiples. Aussi, pour tester l'effet simultané de l'ensemble de ces facteurs ainsi que de leurs interactions sur la VU, un modèle multiple a été construit. Ce modèle inclut les effets principaux de chaque facteur, de même que les interactions possibles d'ordre 2, 3 et 4. Les termes les moins importants ont été ensuite éliminés par une sélection descendante basée sur le test du ratio de vraisemblance (likelihood ratio test) jusqu'à ce que le modèle incluant uniquement les termes significatifs soit retenu (Thomas et al., 2013). Toutes les analyses statistiques ont été conduites dans le logiciel $R$ version 3.5.0 ( $R$ Core Team, 2018) et le seuil de significativité a été fixé à $5 \%$. Pour chaque facteur significatif pour les premiers modèles et pour les modèles finaux obtenus, le pseudo-coefficient de détermination de Nagelkerke (1991) a été calculé dans le package «fmsb» (Nakazawa, 2018) du logiciel $\mathrm{R}$ afin d'examiner la part des variations de $\mathrm{VU}$ expliquée par les facteurs significatifs.

Tableau II.

Noms locaux et significations affectés à Isoberlinia doka et I. tomentosa selon les groupes socioculturels.

\begin{tabular}{|c|c|c|c|}
\hline Espèce & $\begin{array}{l}\text { Groupe } \\
\text { socioculturel }\end{array}$ & Noms locaux & Significations \\
\hline \multirow[t]{8}{*}{ Isoberlinia doka } & Mahi & Kpakpa whlala & Plante à croissance rapide \\
\hline & Nago & Kpakpa odo & Plante aimant les galeries forestières \\
\hline & Anii & Bokoorba & Plante à feuilles blanches \\
\hline & Kotokoli & Kpakpattin & Plante dont les fruits à maturité éclatent dans un bruit sec \\
\hline & Bariba & Bagba & Fruits à maturité éclatant dans un bruit sec \\
\hline & Dendi & Bagba & Éclatements secs des fruits à maturité \\
\hline & Holli & Kpakpa whlala & Plante à croissance rapide \\
\hline & Fon & Kpakpa whlala & Plante à croissance rapide \\
\hline \multirow[t]{8}{*}{ Isoberlinia tomentosa } & Mahi & Kpakpa djéké & Plante à croissance moins rapide \\
\hline & Nago & Kpakpa & Éclatements des fruits à maturité dans un bruit sec \\
\hline & Anii & $\begin{array}{l}\text { Bokoorba } \\
\text { kofoornon }\end{array}$ & Plante à feuilles blanches \\
\hline & Kotokoli & Kpakpattin & Plante dont les fruits à maturité subissent des éclatements secs \\
\hline & Bariba & Bagba & Éclatements secs des fruits à maturité \\
\hline & Dendi & Bagba & Éclatements secs des fruits à maturité \\
\hline & Holli & Kpakpa djéké & Plante à croissance moins rapide \\
\hline & Fon & Kpakpa djéké & Plante à croissance moins rapide \\
\hline
\end{tabular}


Tableau III.

Valeurs propres issues de l'ACP et corrélations entre les usages et les deux premiers axes.

\begin{tabular}{|c|r|r|}
\hline Usages & Axe 1 & Axe 2 \\
\hline $\begin{array}{c}\text { Corrélations } \\
\text { Charpente }\end{array}$ & 0,230 & 0,915 \\
\hline $\begin{array}{c}\text { Fuvre } \\
\text { Énergie }\end{array}$ & $-0,938$ & $-0,071$ \\
\hline Médicinale & 0,636 & $-0,403$ \\
\hline $\begin{array}{c}\text { Valeurs propres issues } \\
\text { de l'ACP et proportions }\end{array}$ & 0,257 & 0,257 \\
Valeurs propres & & \\
\hline \% expliqué & 2,131 & 1,036 \\
\hline \% cumulé & 53,289 & 25,901 \\
\hline
\end{tabular}

\section{Résultats}

\section{Appellations locales de $I$. doka et $I$. tomentosa et leur signification}

Les populations enquêtées connaissent les espèces I. doka et I. tomentosa, les utilisent et les désignent sous diverses appellations selon les groupes socioculturels investigués. Les significations de ces appellations sont surtout liées au mode de croissance, à l'éclatement des fruits, ou à la couleur des feuilles de la plante (tableau II).

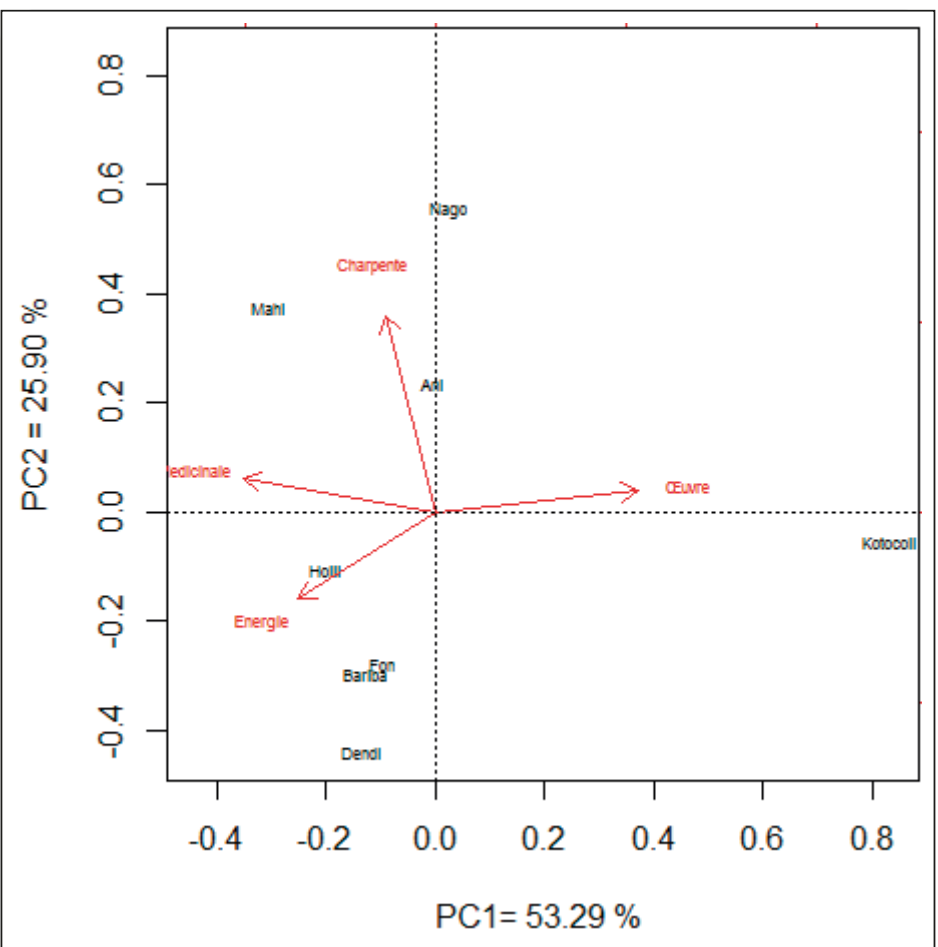

Figure 2.

Répartition des huit groupes socioculturels

utilisant Isoberlinia doka sur le plan factoriel principal de

l'analyse en composantes principales (ACP) des fréquences

relatives de citation (FRC).

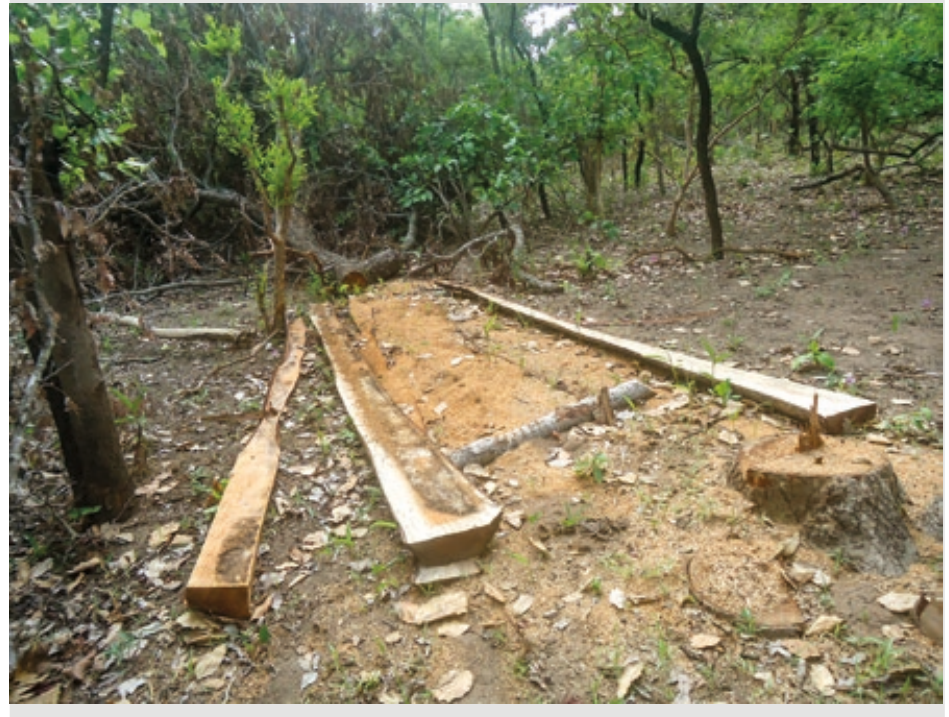

Photo 2.

Extraction des planches de Isoberlinia doka dans la forêt classée de Wari-Maro pour la charpente des maisons.

Photo S. G. C. Adjahossou.

\section{Utilisations de Isoberlinia spp. en fonction des facteurs sociaux}

\section{Patron d'utilisation de I. doka en fonction des groupes socioculturels}

L'analyse en composantes principales (ACP) des valeurs de la FRC des quatre usages par groupe socioculturel a montré que le plan factoriel principal porte 79,2\% des informations liées à l'usage de I. doka (tableau III). Les usages comme bois d'œuvre et bois énergie sont fortement corrélés (|corrélation| $\geq 0,5$ ) avec le premier axe; l'usage pour la charpente est fortement corrélé avec le second axe (tableau III).

La projection des groupes socioculturels sur ces deux premiers axes (figure 2) montre que les Kotocoli utilisent plus I. doka comme bois d'œuvre tandis que les Holli, Bariba, Dendi et Fon l'emploient plus comme bois énergie (photo 1). Les Mahi, Nago et Anii s'intéressent davantage à son utilisation comme bois de charpente (photo 2).

\section{Importance de l'utilisation de I. doka suivant l'âge, la profession, la taille du ménage et le niveau d'instruction}

Les usages importants de $I$. doka sont le bois énergie, le bois de charpente et le bois d'œuvre, avec généralement des fréquences relatives de citation élevées (FRC > 0,5), tandis que, à l'exception du facteur social âge, les usages médicinaux sont moins cités (FRC $<0,3 \%$ ) (figure 3 ). Les personnes adultes sont en général plus concernées par les usages importants (FRC >0,5) (figure 3a). Les charbonniers affichent généralement les plus fortes valeurs de FRC (FRC > 0,98), en comparaison des autres catégories professionnelles (figure $3 \mathrm{~b}$ ). Les non-instruits (FRC >0,7) sont ceux qui en général utilisent le plus l'espèce (figure 3c). Les ménages de grande taille ont une plus grande fréquence relative de citation (figure $3 \mathrm{~d}$ ). 

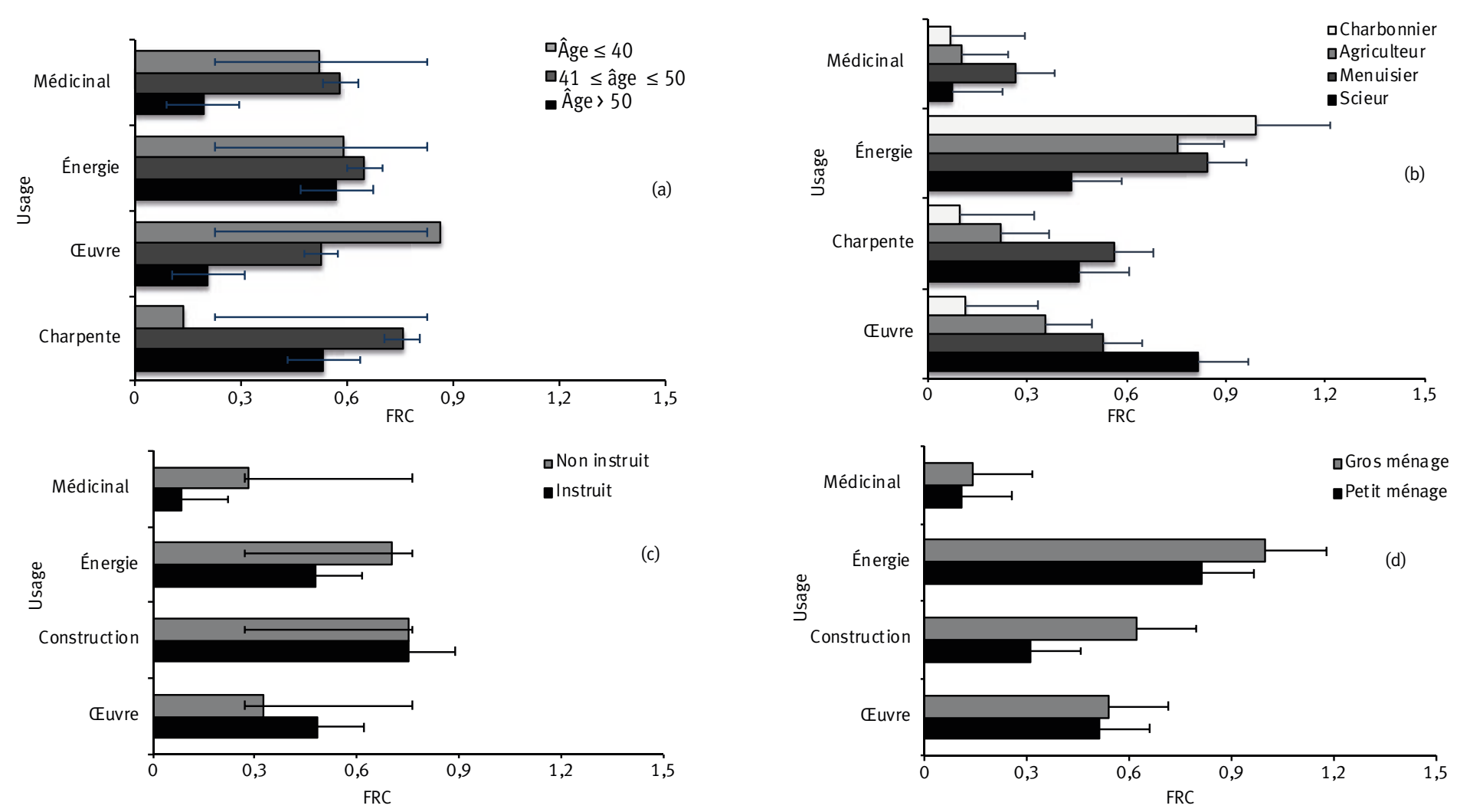

Figure 3.

Utilisations de Isoberlinia doka (FRC \pm erreur type) selon l'âge (a), l'activité socioprofessionnelle (b), le niveau d'instruction (c) et la taille du ménage (d).

\section{Patron d'utilisation de l. tomentosa en fonction des groupes socioculturels}

L'ACP sur les valeurs de la FRC des quatre usages par groupe socioculturel indique que le plan factoriel principal porte $72,4 \%$ des informations liées à l'usage de l. tomentosa (tableau IV). La corrélation des catégories d'usage avec les deux premiers axes (tableau IV) montre que les usages pour le bois d'œuvre et le bois énergie sont fortement corrélés avec le premier axe alors que l'usage pour le bois de charpente et les usages médicinaux sont fortement corrélés avec le second.

La projection des groupes socioculturels sur ces deux premiers axes (figure 4) montre que les Kotokoli, Dendi, Nago, Anii et Mahi ont plus rapporté l'utilisation du bois énergie (photo 3) et d'œuvre (photo 4) tandis que les Bariba ont plus cité l'espèce pour le bois de charpente. Les Holli et Fon utilisent plus l'espèce pour des buts médicinaux, s'agissant notamment de l'utilisation de feuilles, écorces, racines et tiges pour le traitement de douleurs corporelles et de la faiblesse nerveuse ou musculaire des nourrissons.

\section{Importance de l'utilisation de I. tomentosa suivant l'âge,} la profession, le niveau d'instruction et la taille du ménage

Les usages importants de I. tomentosa sont le bois énergie et le bois d'œuvre, avec généralement des fréquences relatives de citation élevées (FRC > 0,50) (figure 5).
Les personnes d'âge élevé (> 40 ans) sont les plus impliquées dans les usages fréquents ( $F R C>0,50$ (figure $5 \mathrm{a}$ ). Les scieurs utilisent en général plus l'espèce pour la catégorie d'usage en charpente. Les autres catégories d'usage

Tableau IV.

Corrélations entre les catégories d'usage et les deux premiers axes

\begin{tabular}{|c|c|c|}
\hline Usages & Axe 1 & Axe 2 \\
\hline $\begin{array}{l}\text { Corrélations } \\
\text { Charpente }\end{array}$ & $-0,324$ & 0,804 \\
\hline Fuvre & 0,796 & $-0,272$ \\
\hline Énergie & 0,863 & 0,223 \\
\hline $\begin{array}{l}\text { Médicinale } \\
\text { Valeurs propres issues } \\
\text { de l'ACP et proportions }\end{array}$ & $-0,324$ & $-0,730$ \\
\hline Valeurs propres & 1,590 & 1,304 \\
\hline \% expliqué & 39,756 & 32,615 \\
\hline \% cumulé & 39,756 & 72,371 \\
\hline
\end{tabular}




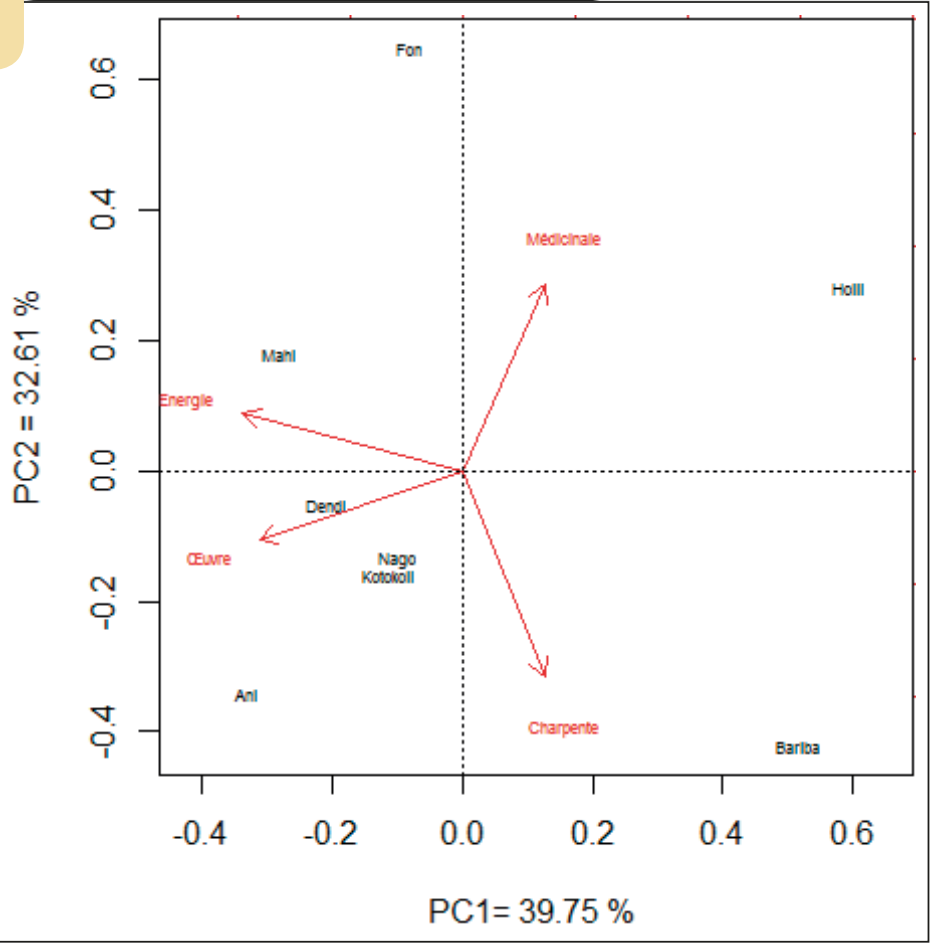

Figure 4.

Répartition des huit groupes socioculturels

utilisant Isoberlinia tomentosa sur le plan factoriel de

l'analyse en composantes principales (ACP) des fréquences relatives de citation (FRC).

sont plutôt plus fréquemment citées par les menuisiers (FRC > 0,55) en comparaison aux autres catégories professionnelles (figure 5b). À l'exception de la catégorie d'usage médicinal ou comme source d'énergie, les personnes instruites utilisent globalement plus l'espèce $(F R C>0,50)$ que les personnes non instruites (figure $5 \mathrm{c}$ ). Les ménages ayant une grande taille ont une fréquence relative de citation plus élevée (figure $5 \mathrm{~d}$ ).

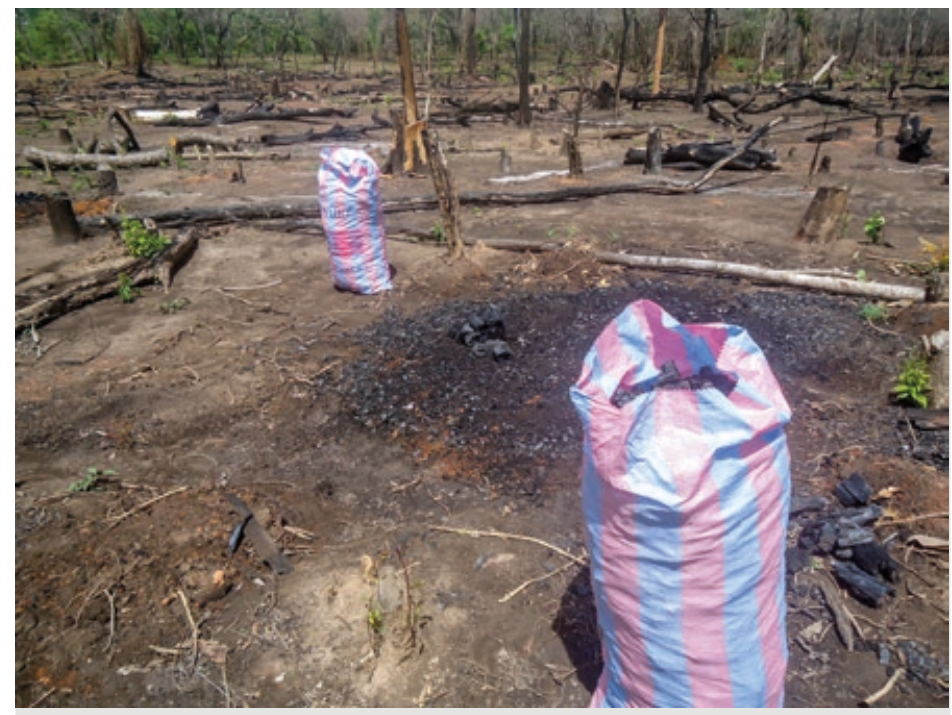

Photo 3.

Charbon de bois à base de Isoberlinia tomentosa et autres espèces ligneuses à Bassila.

Photo S. G. C. Adjahossou.

\section{Valeur d'usage ethnobotanique des essences du genre Isoberlinia en fonction des facteurs sociaux}

\section{Variation des usages de I. doka en fonction des facteurs sociaux}

La valeur d'usage de I. doka varie significativement suivant le groupe socioculturel $\left(p=5,93 \times 10^{-9}\right)$ qui explique environ le tiers des variations de la VU (pseudo- $R^{2}=0,3253$ ). Par contre, aucune différence significative n'est notée, ni suivant l'âge $(p=0,060)$, ni suivant la catégorie professionnelle ( $p=0,360)$, ni suivant le niveau d'instruction $(p=0,542)$ ou la taille du ménage $(p=0,876)$. Le modèle final obtenu après simplification du modèle multiple initial indique également que seul le groupe socioculturel influençait la VU de I. doka. Les Mahi $(2,48 \pm 0,09)$ et les Kotokoli $(2,17 \pm 0,09)$ ont les $\mathrm{VU}$ les plus élevées, alors que les Bariba $(1,07 \pm 0,05)$ et les Holli $(1,42 \pm 0,10)$ ont les VU les plus faibles, les autres groupes ayant des VU intermédiaires.

\section{Variation des usages de I. tomentosa en fonction des facteurs sociaux}

La valeur d'usage de I. tomentosa varie significativement suivant le groupe socioculturel ( $p=0,0005$; pseudo- $\left.R^{2}=0,1605\right)$, la profession ( $p=0,017$; pseudo- $\left.R^{2}=0,640\right)$, et le type de ménage $(p=0,014$; pseudo- $R^{2}=0,376$ ) avec un pouvoir explicatif décroissant tel que l'indiquent les valeurs du coefficient de détermination. Les autres facteurs, notamment l'âge $(p=0,160)$ et le niveau d'instruction ( $p=0,460)$, n'ont aucun effet significatif sur la VU. Le modèle final obtenu après simplification du modèle multiple initial indique que le groupe socioculturel et la profession expliquent ensemble $28,8 \%$ des variations de la VU de I. tomentosa. Les Holli $(2,07 \pm 0,12)$ et les Anii $(2,07 \pm 0,13)$ ont les VU les plus élevées tandis que les Bariba $(1,23 \pm 0,08)$ et les Dendi $(1,35 \pm 0,10)$ ont les VU les plus faibles, les autres groupes ayant des valeurs intermédiaires. Les menuisiers ont la VU la plus élevée $(1,99 \pm 0,11)$ alors que les charbonniers ont la VU la plus faible $(1,33 \pm 0,07)$.

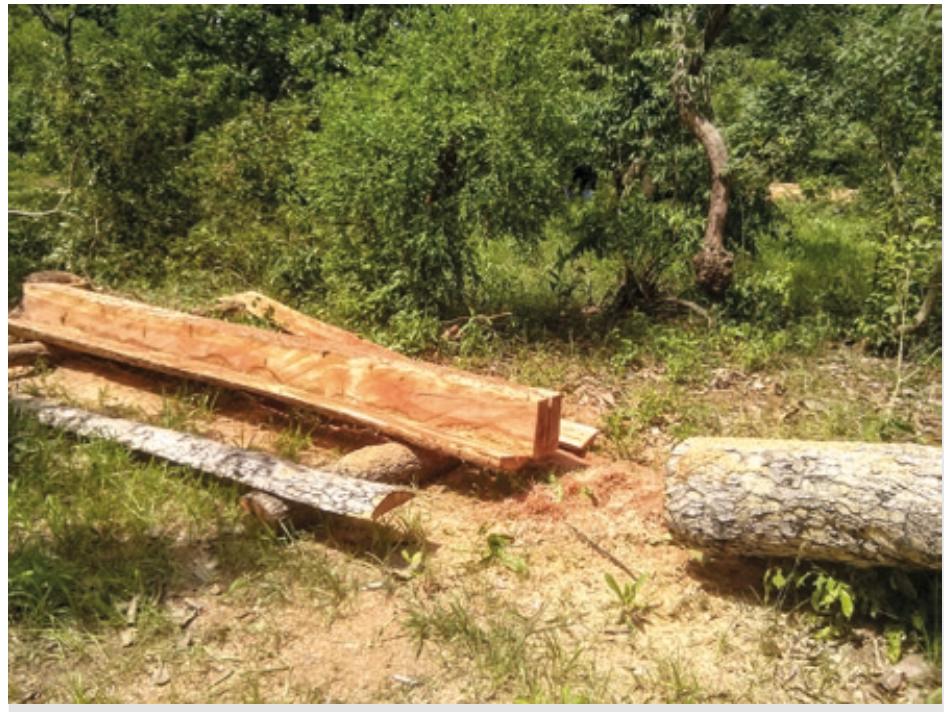

Photo 4.

Tronc de Isoberlinia tomentosa façonné en madrier dans la forêt classée de Wari-Maro.

Photo S. G. C. Adjahossou. 

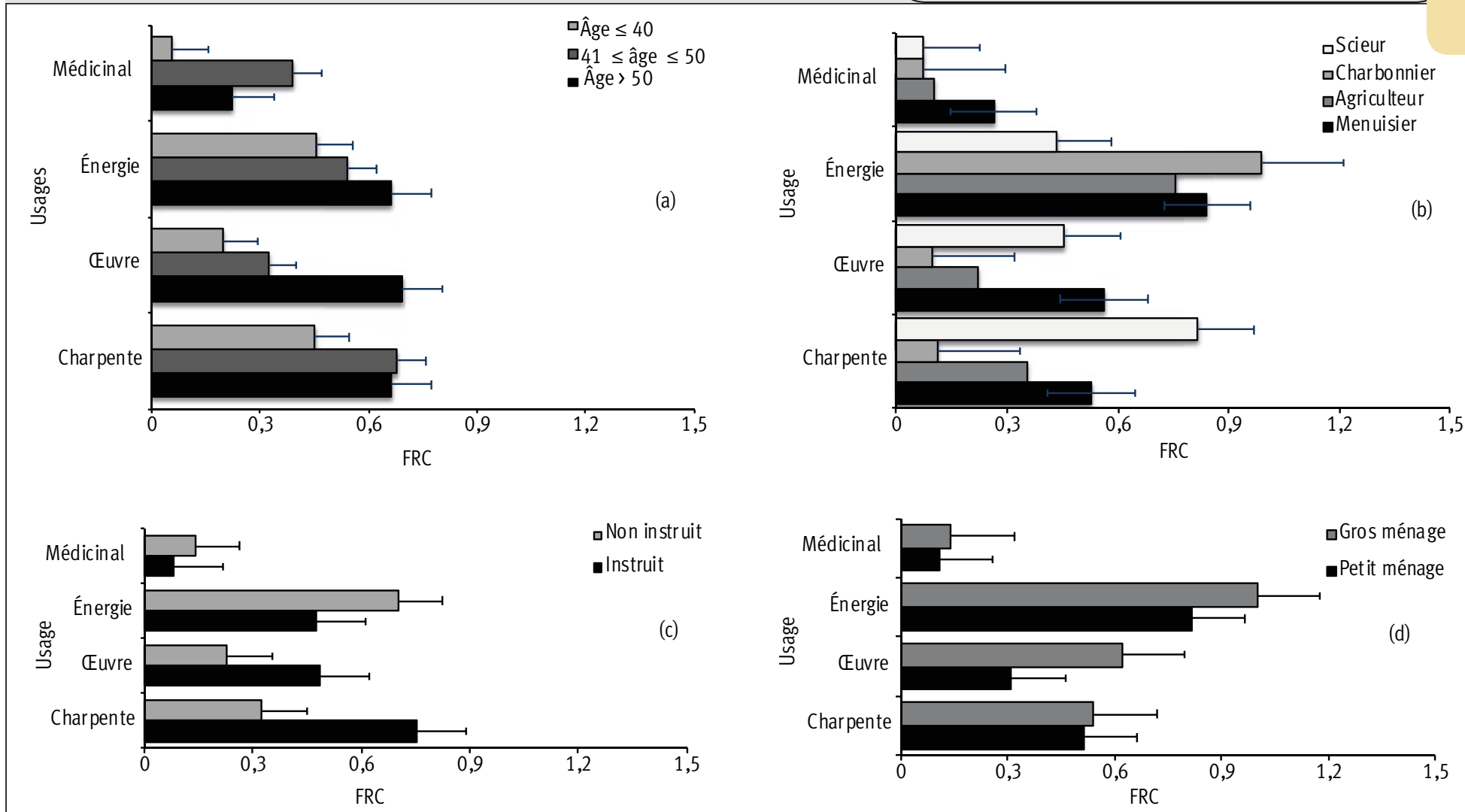

Figure 5.

Utilisations de Isoberlinia tomentosa (FRC \pm erreur type) selon l'âge (a), l'activité socioprofessionnelle (b), le niveau d'instruction (c) et la taille du ménage (d).

\section{Discussion}

\section{Variation de l'usage du genre Isoberlinia suivant les facteurs sociaux}

L'étude a permis de recenser les utilisations des deux essences réparties en quatre catégories d'usage, à savoir le bois d'œuvre, le bois de charpente, le bois énergie et l'usage médicinal. Les résultats renseignent le degré de diversité des usages des deux essences dans le Moyen-Bénin et corroborent les résultats de Dourma et al. (2009) au Togo, où ces espèces assurent des fonctions déterminantes dans la vie quotidienne des populations locales. Les différences statistiques significatives des valeurs d'usage entre les groupes socioculturels montrent que l'importance culturelle des espèces varie suivant ces groupes socioculturels, et suggèrent une diversité importante et une spécification des usages au sein des populations locales. Ces spécificités pourraient résulter des perceptions séculaires, des modes de vie et des activités professionnelles des groupes socioculturels (Houéhanou et al., 2011 ; Sinasson et al., 2017). En effet, les Mahi préfèrent $l$. doka pour le bois d'œuvre comparativement aux autres groupes socioculturels alors que les Kotocoli utilisent plus la même espèce pour des besoins de charpente. Généralement, dans le milieu d'étude, les Mahi et les Fon font partir des groupes socioculturels qui se convertissent le plus dans les activités du bois (menuiserie, exploitants forestiers, scieurs et charbonniers), ceci à cause de la rareté des terres cultivables et la baisse de devises agricoles (Adjahossou et al., 2018). En conséquence, ces groupes ethniques détiennent plus de connaissances liées aux usages du bois. Par ailleurs, il y a une affinité plus grande pour le bois blanc de I. tomentosa qui est plus dur que pour celui de I. doka qui est moins dur.

Les modèles simples à un facteur montrent que, parmi les facteurs testés, seul le groupe socioculturel détermine à la fois les variations de l'usage des deux essences ; ils témoignent d'une forte concentration des connaissances de I. tomentosa au sein des Holli et Anii et de I. doka au sein des Mahi et Kotokoli. Ces résultats soutiennent l'hypothèse de connaissances des plantes dépendantes des groupes socioculturels (Houéhanou et al., 2011).

La catégorie socioprofessionnelle se révèle également un facteur important dans la spécification des usages de $l$. tomentosa. Par exemple, les menuisiers détiennent plus de connaissances sur sa bonne qualité technologique et, par conséquent, l'utilisent plus pour le bois d'œuvre. Quant aux Anii, ils l'utilisent surtout comme bois énergie alors que les Holli et Fon emploient préférentiellement l'écorce, la racine et les jeunes plants entiers pour le traitement de diverses affections. Les fréquents prélèvements de racines et écorces exposeraient $I$. doka à des risques de vulnérabilité (Betti, 2001 ; Traoré et al., 2011 ; Badjaré et al., 2018). Le penchant des Holli et Fon vers les usages médicinaux de I. tomentosa 
peut s'expliquer par la disponibilité de la ressource dans leur milieu selon l'hypothèse de l'apparence écologique (Albuquerque, 2006). Aussi les ligneux médicinaux les plus prisés tels que Khaya senegalensis et Afzelia africana sontils devenus rares dans leurs terroirs. Les usages médicinaux recensés dans cette étude corroborent les nombreux travaux ethnobotaniques déjà réalisés en Afrique sur le genre Isoberlinia. Ces travaux ont montré qu'en Côte d'Ivoire, l'écorce de I. tomentosa sert à traiter les fièvres ainsi que les douleurs corporelles (Ahoba et al., 1995). Au Togo, la macération d'écorce s'utilise pour soigner les lésions de la peau ou comme vermifuge (Dourma et al., 2009) alors qu'au Mali l'infusion de feuilles est utilisée en lavage par les femmes contre la stérilité (Louppe, 2012).

Les modèles multiples des effets principaux des cinq facteurs sociaux investigués sur la variation de la valeur d'usage de l. tomentosa montrent des interactions significatives (Thomas et al., 2013) entre le groupe socioculturel et l'activité professionnelle. Ce résultat suggère que les différences entre les groupes ethniques peuvent être amplifiées par l'activité socioprofessionnelle. Par exemple, l'importance culturelle accordée par les Holli et Kotokoli à I. tomentosa sur le plan médicinal est influencée significativement quand ces derniers sont de la catégorie socioprofessionnelle des menuisiers et charbonniers et ceci réciproquement.

Les appellations locales spécifiques des groupes socioculturels relatives aux essences ligneuses dépendent aussi de ces groupes socioculturels et sont liées au vécu quotidien de chaque groupe avec ces arbres (Camou-Guerrero et al., 2008).

\section{Conclusion et implications}

Le genre Isoberlinia participe à la satisfaction de multiples besoins en bois au Moyen-Bénin. Le test des effets principaux des facteurs sociaux pris isolément montre que la valeur d'usage varie significativement suivant le groupe socioculturel pour 1 . doka et suivant l'activité professionnelle, la taille du ménage et le groupe socioculturel pour I. tomentosa. L'évaluation des interactions suggère que le groupe socioculturel constitue le facteur le plus déterminant de la valeur d'usage totale de $I$. doka tandis que pour $I$. tomentosa, en plus du groupe socioculturel, s'ajoute l'activité professionnelle.

Nous suggérons la vulgarisation de stratégies d'exploitation durable des deux espèces au sein des groupes socioculturels. Ainsi, des méthodes durables de récolte d'organes des deux essences peuvent être vulgarisées au sein des Fon et Holli. La promotion de la plantation d'espèces alternatives pour les usages basés sur le bois peut être envisagée au sein des groupes sociaux culturels et professionnels ayant une inclination pour les usages du bois des deux espèces étudiées. Au regard du potentiel des essences du genre Isoberlinia pour la satisfaction des besoins relatifs au bois, la maîtrise des structures spatiales et la sylviculture constituent des contraintes à lever pour leur intégration dans les opérations d'enrichissement de forêts claires et plantations locales. Ceci nécessite toutefois l'acquisition de connaissances scientifiques sur les techniques de multiplication végétative, peu onéreuses, afin de collecter les meilleurs clones vis-à-vis des demandes privilégiées des exploitants forestiers.

\section{Remerciements}

Cette étude a été réalisée grâce aux fonds du Gouvernement béninois à travers l'allocation du ministère de l'Enseignement supérieur et de la Recherche scientifique (MESRS) dans le cadre de l'initiative "Appui aux doctorants ». Les auteurs remercient Joël Adandonon pour son assistance aux analyses statistiques de même que l'assistant de recherche Pathmos Akouete, et enfin Idelphonse Glegbeto et Ruffin Kpatinnou pour l'aide précieuse apportée lors de la collecte de nos données de terrain.

\section{Références bibliographiques}

Adjahossou S. G. C., Houéhanou D. T., Toyi M., Tente B., Houinato M., Sinsin B., 2018. Degré de pression et perception endogène de multiplication et de conservation du genre Isoberlinia au Moyen-Bénin (Afrique de l'Ouest). Science et Technique, Sciences Naturelles et Appliquées, 4 (1) : 25-38.

Adjahossou S. G. C., Houéhanou D. T., Sodé A. S., Houinato M. R. B., Sinsin B., 2016. Efficacité des aires protégées dans la conservation d'habitats favorables prioritaires de ligneux de valeur au Bénin. Bois et Forêts des Tropiques, 328 (2) : 67-76. https://doi. org $/ 10.19182 / \mathrm{bft} 2016.328 . a 31303$

Ahoba A., Edi K., Diby K., 1995. Propriétés technologiques et possibilités d'utilisation de sept essences de savane de Côte d'Ivoire. Abidjan, Côte d'Ivoire, IDEFOR/DFO, 27 p.

Ahoyo C. C., Houéhanou T. D., Yaoitcha A. S., Prinz K., Assogbadjo A. E., Adjahossou S. G. C., Hellwig F., Houinato M. R. B., 2018. A quantitative ethnobotanical approach toward biodiversity conservation of useful woody species in Wari Maro forest reserve (Benin, West Africa). Environment, Development and Sustainability, 20 (5): 2301-2320.

Akouègninou A., Van der Burg W. J., Van der Maesen L. J. G., Adjakidjè V., Essou J. P., Sinsin B., Yèdomanhan H., 2006. Flore analytique du Bénin. Leiden, Pays-Bas, Backhuys Publishers, 1034 p.

Akpona J. D. T., Assogbadjo A. E., Fandohan A. B., Kakaï R. G., 2017. Inventaire et approche multicritères pour identifier les essences prioritaires pour la conservation au Bénin. Bois et Forêts des Tropiques, 333 (3) : 5-16. https://doi.org/10.19182/bft2017.333. a31461

Albuquerque U. P., 2006. Re-examining hypotheses concerning the use and knowledge of medicinal plants: A study in the Caatinga vegetation of NE Brazil. Journal of Ethnobiology and Ethnomedicine, 2 (1): 30.

Assogba G. A., Fandohan A. B., Salako V. K., Assogbadjo A. E., 2017. Usages de Bombax costatum (Malvaceae) dans les terroirs riverains de la Réserve de biosphère de la Pendjari, République du Bénin. Bois et Forêts des Tropiques, 333 (3) : 17-33. http://dx.doi. org/10.19182/bft2017.333.a31465

Assogbadjo A. E., Glèlè Kakaï R., Chadaré F. J., Thomson L., Kyndt T., Sinsin B., Van Damme P., 2008. Folk classification, perception, and preferences of baobab products in West Africa: consequences for species conservation and improvement. Economic Botany, 62 (1): 74-84.

Badjaré B., Kokou K., Bigou-laré N., Koumantiga D., Akpakouma A., Adjayi M. B., Abbey G. A., 2018. Étude ethnobotanique d'espèces ligneuses des savanes sèches au Nord-Togo : diversité, usages, importance et vulnérabilité. Biotechnology, Agronomy, Society and Environment, $22: 2-20$. 
Bationo B. A., Ouedraogo S. J., Somé A. N., Pallo F., Boussim I. J., 2005. Régénération naturelle de $I$. doka dans la forêt classée du Nazinon (Burkina Faso). Cahiers Agricultures, 14 (3) : 297-304.

Bellefontaine R., Meunier Q., Ichaou A., Le Bouler H., 2015. Multiplication végétative à faible coût au profit des paysans et éleveurs des zones tropicales et méditerranénnes. Vertigo - La Revue Électronique en Sciences de l'Environnement. http://vertigo.revues. org/16516

Betti J. L., 2001. Vulnérabilité des plantes utilisées comme antipaludiques dans l'arrondissement de Mintom au sud de la réserve de biosphère du Dja (Cameroun). Systematics and Geography of Plants, 71: 661-678.

Camou-Guerrero A., Reyes-Garcia., Martinez-Ramos M., Cassa A., 2008. Knowledge and use value of plants species in a Raramuri community: a gender perspective for conservation. Human Ecology, 36: 259-272. https://link.springer.com/article/10.1007/ $\underline{\mathrm{s} 10745-007-9152-3}$

Dagnelie P., 1998. Statistiques théoriques et appliquées. Bruxelles, Belgique, De Boeck Services, 517 p.

Dourma M., 2011. Les forêts claires à Isoberlinia doka Craib \& Stapf et I. tomentosa (Harms) Craib \& Stapf (Fabaceae) en zone soudanienne du Togo : écologie, régénération naturelle et activités humaines. Résumé de thèse d’État. Acta Botanica Gallica, 158 (1) : 141-144, 201.

Dourma M., Wala K., Bellefontaine R., Batawila K., Guelly A. K., Akpagana K., 2009. Comparaison de l'utilisation des ressources forestières et de la régénération entre deux types de forêts claires à Isoberlinia au Togo. Bois et Forêts des Tropiques, 302 (4) : 5-19. http://revues.cirad.fr/index.php/BFT/article/view/20400

Etongo D., Djenontin I. N. S., Kanninen M., Glover E. K., 2017. Assessing use-values and relative importance of trees for livelihood values and their potentials for environmental protection in Southern Burkina Faso. Environment, Development and Sustainability, 19: 1141-1166.

Fandohan A. B., Chadare F. J., Gouwakinnou G. N., Tovissode C. F., Bonou A., Djonlonkou S. F. B., Houndelo L. F. H., Sinsin C. L. B., Assogbadjo A. E., 2017. Usages traditionnels et valeur économique de Synsepalum dulcificum au Sud-Bénin. Bois et Forêts des Tropiques, $332(2): 17-30$

Fandohan A. B., Gouwakinnou N. G., Déléké Koko K. I. E., Glèlè Kakaï R., Assogbadjo A. E., 2015. Domesticating and conserving indigenous trees species: an ecosystem based approach for adaptation to climate change in Sub-Sahara Africa. CAMES, 3 (1): 55-60.

Fandohan B. A., Assogbadjo A. E., Glèlè Kakaï R., Kyndt T., de Caluwé T. E., Codjia J. T. C., Sinsin B., 2010. Women's Traditional Knowledge, Use Value, and the Contribution of Tamarind (Tamarindus indica L.) to Rural Households' Cash Income in Benin. Economic Botany, 64 (3): 248-259. https://doi.org/10.19182/bft2009.302.a20400

Gavin M. C., Anderson G. J., 2017. Socioeconomic predictors of forest use values in the Peruvian Amazon: A potential tool for biodiversity conservation. Ecological Economics, 60 (1): 752-762.

Gouwakinnou G. N., Lykke A. M., Assogbadjo A. E., Sinsin B., 2011. Local knowledge, pattern and diversity of use of Sclerocarya birrea. Journal of Ethnobiology and Ethnomedicine, 7 (1): 1-9.

Houéhanou D. T., Assogbadjo A. E., Chadare F. J., Zanvo S., Sinsin B., 2016. Approches méthodologiques synthétisées des études d'ethnobotanique quantitative en milieu tropical. Annales des Sciences Agronomiques, $20: 187-205$.

Houéhanou T. D., Assogbadjo A. E., Glèlè Kakaï R., Houinato M., Sinsin B., 2011. Valuation of local preferred uses and traditional ecological knowledge in relation to three multipurpose tree species in Benin (West Africa). Forest Policy and Economics, 13: 554-562.

INSAE, 2016. Rapport du Quatrième Recensement Général de la Population et de l'Habitation. Résultats : caractéristiques générales de la population. Cotonou, Bénin, INSAE, 8 p.

Levy P. S., Lemeshow S., 2008. Sampling of populations: Methods and Applications. New York, USA, Wiley, 192 p.
Lougbégnon T. O., Tente B. A. H., Amontcha M., Codjia J. T. C., 2011. Importance culturelle et valeur d'usage des ressources végétales de la réserve forestière marécageuse de la vallée de Sitatunga et zones connexes. Bulletin de la Recherche Agronomique du Bénin, $70: 35-46$.

Louppe D., 2012. Isoberlinia doka Craib \& Stapf. In : Lemmens R. H. M. J., Louppe D., Oteng-Amoako A. A. (éds). Ressources végétales de l'Afrique tropicale. PROTA 7 (2). Bois d'œuvre 2. Wageningen, PaysBas, Fondation PROTA, 465-470. http://www.prota4u.org/search. asp

Nakazawa M., 2018. fmsb: Functions for Medical Statistics Book with some Demographic Data. R package version 0.6.3. https:// CRAN.R-project.org/package $=$ fmsb

Nagelkerke N. J. D., 1991. A Note on a General Definition of the Coefficient of Determination. Biometrika, 78 (3): 691-692. https://www. jstor.org/stable/i315532

Oloukoï J., 2012. Utilité de la télédétection et des Systèmes d'Information Géographique dans l'étude de la dynamique spatiale de l'occupation des terres au Centre du Bénin. Thèse de doctorat unique, FLASH/UAC, Bénin, 304 p.

Phillips O., Gentry A. H., 1993. The useful plants of Tambopata, Peru: Additional hypothesis testing in quantitative ethnobotany. Economy Botany, 47: 33-43.

R Core Team, 2018. R: A language and environment for statistical computing. Vienna, Austria, R Foundation for Statistical Computing. https://www.R-project.org/

Salako K. V., Moreira F., Gbedomon R. C., Tovissodé F., Assogbadjo A. E., Kakaï R. L. G., 2018. Traditional knowledge and cultural importance of Borassus aethiopum Mart. in Benin: interacting effects of socio-demographic attributes and multi-scale abundance. Journal of Ethnobiology and Ethnomedicine, 14 (1): 36.

Sinasson G. K. S., Shackleton C. M., Assogbadjo A. E., Sinsin B., 2017. Local knowledge on the uses, habitat, and change in abundance of multipurpose Mimusops species in Benin. Economic Botany, 20 (10): 1-18.

Souto T., Ticktin T., 2012. Understanding interrelationships among predictors (age, gender, and origin) of local ecological knowledge. Economic Botany, 66 (2): 149-164.

Tardío J., Pardo-de-Santayana M., 2008. Cultural Importance Indices: A Comparative Analysis Based on the Useful Wild Plants of Southern Cantabria (Northern Spain). Economic Botany, 62: 24-39.

Thomas R., Vaughan I., Lello J., 2013. Data analysis with R statistical software. A guidebook for scientists. 1st edition. Cardiff, United Kingdom, Eco-explore $\mathrm{CIC}$.

Traoré L., Ouédraogo I., Ouédraogo A., Thiombiano A., 2011. Perceptions, usages et vulnérabilité des ressources végétales ligneuses dans le Sud-Ouest du Burkina Faso. International Journal of Biological and Chemical Sciences, 5 : 258-278.

Yabi I., 2007. Étude de l'agroforesterie à base de l'anacardier et des contraintes climatiques à son développement dans le Centre du Bénin. Thèse de doctorat unique, FLASH/UAC, Bénin, 239 p.

Yaoitcha A. S., Houéhanou T. D., Fandohan A. B., Houinato M. R. B., 2015. Prioritization of useful medicinal tree species for conservation in Wari-Maro Forest Reserve in Benin: A multivariate analysis approach. Forest Policy and Economics, 61 (2): 135-146.

Bois et Forêts des Tropiques - Revue scientifique du Cirad

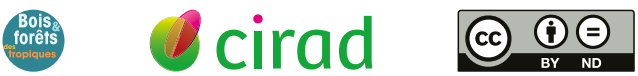

Cirad - Campus international de Baillarguet, 34398 Montpellier Cedex 5, France - Contact : bft@cirad.fr - ISSN : L-0006-579X 